\title{
ERRATUM
}

\section{Erratum to: Loss of heterozygosity analysis in malignant gliomas}

\author{
Masahiro Mizoguchi • Daisuke Kuga • \\ Yanlei Guan • Nobuhiro Hata • Akira Nakamizo • \\ Koji Yoshimoto · Tomio Sasaki
}

Published online: 13 July 2011

(C) The Japan Society of Brain Tumor Pathology 2011

\section{Erratum to: Brain Tumor Pathol}

\section{DOI 10.1007/s10014-011-0038-0}

Unfortunately, in Table 1 of the article cited above, the 3rd line in the first column appeared incorrectly.

Table 1 should be correctly shown as follows:

Table 1 Comparison of common molecular assays for $\mathrm{LOH}$ detection

\begin{tabular}{lllll}
\hline & $\begin{array}{l}\text { PCR- } \\
\text { based } \\
\text { assay }\end{array}$ & FISH & $\begin{array}{l}\text { CGH } \\
(\mathrm{aCGH})\end{array}$ & $\begin{array}{l}\text { SNP } \\
\text { array }\end{array}$ \\
\hline Allelic imbalance & $\bigcirc$ & $\times$ & $\times$ & $\bigcirc$ \\
$\begin{array}{l}\text { Copy number alterations } \\
\text { Copy neutral LOH (UPD) } \\
\text { (mitotic recombination) }\end{array}$ & $\bigcirc$ & $\times$ & $\times$ & $\bigcirc$ \\
Homozygous deletions & $\times$ & $\bigcirc$ & $\bigcirc$ & $\bigcirc$ \\
Matching DNA required & $\bigcirc$ & $\times$ & $\bigcirc$ & $\Delta^{\mathrm{a}}$ \\
\hline
\end{tabular}

$\overline{{ }^{a} \text { High resolution SNP array can detect } \mathrm{LOH} \text { regions without }}$ matching DNA

The online version of the original article can be found under doi:10.1007/s10014-011-0038-0.

M. Mizoguchi $(\varangle) \cdot$ D. Kuga · Y. Guan · N. Hata ·

A. Nakamizo · K. Yoshimoto · T. Sasaki

Department of Neurosurgery, Graduate School of Medical

Sciences, Kyushu University, 3-1-1 Maidashi, Higashi-ku,

Fukuoka 812-8582, Japan

e-mail: mmizoguc@ns.med.kyushu-u.ac.jp 